\title{
EIGENVALUES OF THE NEGATIVE LAPLACIAN FOR ARBITRARY MULTIPLY CONNECTED DOMAINS
}

\author{
E. M. E. ZAYED \\ Mathematics Department \\ Faculty of Science \\ Zagazig University \\ Zagazig, EGYPT
}

(Received October 6, 1994 and in revised form January 17, 1995)

ABSTRACT. The purpose of this paper is to derive some interesting asymptotic formulae for spectra of arbitrary multiply connected bounded domains in two or three dimensions, linked with variation of positive distinct functions entering the boundary conditions, using the spectral function $\sum_{k=1}^{\infty}\left\{\mu_{k}\left(\sigma_{1}, \ldots, \sigma_{n}\right)+P\right\}^{-2}$ as $P \rightarrow \infty$. Further results may be obtained.

KEY WORDS AND PHRASES. Inverse problem, negative Laplacian, eigenvalues, spectral functions. 1991 AMS SUBJECT CLASSIFICATION CODES. 35K, 35P.

\section{INTRODUCTION.}

The underlying inverse eigenvalue problem (1.1)-(1.2) has been discussed recently by Zayed [1] and has shown that some geometric quantities associated with a bounded domain can be found from a complete knowledge of the eigenvalues $\left\{\mu_{k}(\sigma)\right\}_{k=1}^{\infty}$ for the negative Laplacian $-\triangle_{n}=-\sum_{i=1}^{n}\left(\frac{\partial}{\partial x^{i}}\right)^{2}$ in $R^{n}(n=2$ or 3$)$.

Let $\Omega$ be a simply connected bounded domain in $R^{n}$ with a smooth boundary $\partial \Omega$ in the case $n=2$ (or a smooth bounding surface $S$ in the case $n=3$ ). Consider the impedance problem

$$
\begin{aligned}
&-\triangle_{\mathrm{n}} \mathrm{u}=\lambda \mathrm{u} \text { in } \quad \Omega, \\
&\left(\frac{\partial}{\partial n}+\sigma\right) u=0 \quad \text { on } \quad \partial \Omega \quad(\text { or } S),
\end{aligned}
$$

where $\frac{\partial}{\partial n}$ denotes differentiation along the inward pointing normal to $\partial \Omega$ or $S$, and $\sigma$ is a positive function.

Denote its eigenvalues, counted according to multiplicity, by

$$
0<\mu_{1}(\sigma) \leq \mu_{2}(\sigma) \leq \ldots \leq \mu_{k}(\sigma) \leq \ldots \rightarrow \infty \quad \text { as } \quad k \rightarrow \infty .
$$

It is well known [2] that in the case $n=2$

$$
\mu_{k}(\sigma) \sim\left(\frac{4 \pi}{|\Omega|}\right) k \quad \text { as } \quad k \rightarrow \infty
$$

while in the case $n=3$ 


$$
\mu_{k}(\sigma) \sim\left(\frac{6 \pi^{2}}{V} k\right)^{2 / 3} \quad \text { as } \quad k \rightarrow \infty,
$$

where $|\Omega|$ and $V$ are respectively the area and the volume of $\Omega$.

The purpose of this paper is to discuss the following more general inverse problem: Let $\Omega$ be an arbitrary multiply connected bounded domain in $R^{n}(n=2$ or 3$)$ which is surrounded internally by simply connected bounded domains $\Omega_{\imath}$ with smooth boundaries $\partial \Omega_{\imath}$ in the case $n=2$ (or smooth bounding surfaces $S_{\imath}$ in the case $n=3$ ) where $i=1,2, \ldots, m-1$, and externally by a simply connected bounded domain $\Omega_{m}$ with a smooth boundary $\partial \Omega_{m}$ in the case $n=2$ (or a smooth bounding surface $S_{m}$ in the case $n=3$ ). Suppose that the eigenvalues

$$
0<\mu_{1}\left(\sigma_{1}, \ldots, \sigma_{m}\right) \leq \mu_{2}\left(\sigma_{1}, \ldots, \sigma_{m}\right) \leq \ldots \leq \mu_{k}\left(\sigma_{1}, \ldots, \sigma_{m}\right) \leq \ldots \longrightarrow \infty \text { as } k \rightarrow \infty
$$

are known exactly for the impedance problem

$$
\begin{gathered}
-\triangle_{n} u=\lambda u \quad \text { in } \quad \Omega, \\
\left(\frac{\partial}{\partial n_{\imath}}+\sigma_{\imath}\right) u=0 \quad \text { on } \quad \partial \Omega_{i} \quad \text { (or } S_{i} \text { ), }
\end{gathered}
$$

where $\frac{\partial}{\partial m_{i}}$ denote differentiations along the inward pointing normals to $\partial \Omega_{\imath}$ or $S_{\boldsymbol{i}}$ respectively and $\sigma_{i}$ are positive functions $(i=1, \ldots, m)$.

In Theorem 2.1, we determine some geometric quantities associated with the multiply connected domain $\Omega$ from the complete knowledge of the eigenvalues (1.6) for the problem (1.7)-(1.8) using the asymptotic expansion of the spectral function

$$
\sum_{k=1}^{\infty} \frac{1}{\left[\mu_{k}\left(\sigma_{1}, \ldots, \sigma_{m}\right)+P\right]^{2}} \quad \text { as } \quad P \rightarrow \infty
$$

where $P$ is a positive constant, while $\sigma_{i}$ are positive functions defined on $\partial \Omega_{i}$ or $S_{i}(i=1, \ldots, m)$ and satisfying the Lipschitz condition.

In Theorem 2.2, we show that the asymptotic expansion of (1.9) as $P \rightarrow \infty$ plays an important role in establishing a method to study the asymptotic behavior of the difference

$$
\sum_{0<\mu_{k}\left(\sigma_{1}, \ldots, \sigma_{m}\right) \leq \lambda}\left[\mu_{k}\left(\alpha_{1}, \ldots, \alpha_{m}\right)-\mu_{k}\left(\beta_{1}, \ldots, \beta_{m}\right)\right] \quad \text { as } \quad \lambda \rightarrow \infty,
$$

where $\sigma_{\imath}(Q), \alpha_{i}(Q), \beta_{\imath}(Q) Q \in \partial \Omega_{\imath}$ (or $\left.Q \in S_{i}\right),(i=1, \ldots, m)$ are, generally speaking, distinct functions and satisfying the Lipschitz condition and the summation is taken over all values of $k$ for which $\mu_{k}\left(\sigma_{1}, \ldots, \sigma_{m}\right) \leq \lambda$.

Note that theorems and corollaries of this paper contain further results similar to those obtained recently by Zayed and Younis [2].

\section{STATEMENT OF RESULTS}

Using methods similar to those obtained in [1], [2], we can easily prove the following theorems:

THEOREM 2.1. If the functions $\sigma_{\imath}(Q), Q \in \partial \Omega_{\imath}$ (or $Q \in S_{\imath}$ ), $(i=1, \ldots, m$ ) satisfy the Lipschitz condition, then in the case $n=2$ 


$$
\begin{aligned}
& \sum_{h}^{x} \frac{1}{\left[\mu_{h}\left(\sigma_{1}, \ldots, \sigma_{m}\right)+P\right]^{2}}=\frac{|\Omega|}{4 \pi P}+\left(\sum_{1}^{m} L_{1}\right) / 16 P^{32} \\
& +\frac{1}{6 P^{2}}\left\{(2-m)+\frac{3}{\pi}\left[\sum_{1}^{m} \int_{\partial \Omega_{2} .} \sigma_{1}(Q) d Q-2 \sum_{1}^{m} \int_{\partial S_{2}} \sigma_{,}(Q) d Q\right]\right\} \\
& +\frac{21}{1024 P^{5 \cdot 2}} \sum_{1}^{m} \int_{\partial \Omega_{2} .}\left\{K_{,}^{2}(Q)-\frac{32}{7}\left[\sigma_{,}(Q) K_{1}(Q)\right.\right. \\
& \left.\left.-2 \sigma_{,}^{2}(Q)\right]\right\} d Q+0\left(\frac{1}{P^{3}}\right) \quad \text { as } \quad P \rightarrow \infty,
\end{aligned}
$$

where $L$, and $K_{\imath}(Q)(\imath=1, \ldots, m)$ are respectively the total lengths and the curvatures of $\partial \Omega$, at the point $Q$, while in the case $n=3$ we deduce that

$$
\begin{aligned}
\sum_{k=1}^{x} \frac{1}{\left[\mu_{k}\left(\sigma_{1}, \ldots, \sigma_{m}\right)+P\right]^{2}} & =\frac{V}{8 \pi P^{1^{\prime 2}}}+\left(\sum_{\imath=1}^{m}\left|S_{\imath}\right|\right) / 16 \pi P \\
& +\frac{1}{24 \pi P^{32}} \sum_{\imath=1}^{m} \int_{S_{\imath}}\left[H_{\imath}(Q)-3 \sigma_{\imath}(Q)\right] d Q \\
& +\frac{7}{128 \pi P^{2}} \sum_{\imath=1}^{m} \int_{S_{i}}\left\{\left[H_{\imath}(Q)-3 \sigma_{\imath}(Q)\right]^{2}\right. \\
& \left.-\left[N_{\imath}(Q)-\frac{26}{7} \sigma_{\imath}(Q) H_{\imath}(Q)+\frac{47}{7} \sigma_{\imath}^{2}(Q)\right]\right\} d Q \\
& +\frac{39}{5760 \pi P^{5 / 2}} \sum_{\imath=1}^{m} \int_{S_{\imath}}\left[H_{\imath}(Q)-3 \sigma_{\imath}(Q)\right]^{3} d Q \\
& +0\left(\frac{1}{P^{3}}\right) \quad \text { as } \quad P \rightarrow \infty,
\end{aligned}
$$

where $\left|S_{\imath}\right|, H_{\imath}(Q)$ and $N_{\imath}(Q)$ are respectively the surface areas, mean curvatures and Gaussian curvatures of the bounding surfaces $S_{2}(i=1, \ldots, m)$

Formulae (2 1) and (2 2) can be considered as a generalization of the formula (2 3 ) obtained by Zayed [3] and the formula (2 3) obtained by Zayed [4] respectively

THEOREM 2.2. If the functions $\sigma_{\imath}(Q), \alpha_{\imath}(Q), \beta_{\imath}(Q), Q \in \partial \Omega_{\imath}$ (or $\left.Q \in S_{\imath}\right)(i=1, \ldots, m$ ) are distinct and satisfy the Lipschitz condition, then we deduce for $\lambda \rightarrow \infty$ that

$$
\sum_{\mu_{k}\left(\sigma_{1}, \sigma_{m}\right) \leq \lambda}\left[\mu_{k}\left(\alpha_{1}, \ldots, \alpha_{m}\right)-\mu_{k}\left(\beta_{1}, \ldots, \beta_{m}\right)\right]=\left\{\begin{array}{l}
{\left[\frac{a_{1}}{2 \pi}+o(1)\right] \lambda \quad \text { in the case } n=2,} \\
{\left[\frac{b_{1}}{3 \pi^{2}}+o(1)\right] \lambda^{3 / 2} \text { in the case } n=3,}
\end{array}\right.
$$

where

$$
a_{1}=2 \sum_{\imath=2}^{m} \int_{\partial \Omega_{\imath}}\left[\alpha_{\imath}(Q)-\beta_{\imath}(Q)\right] d Q-\sum_{\imath=1}^{m} \int_{\partial \Omega_{\imath}}\left[\alpha_{\imath}(Q)-\beta_{\imath}(Q)\right] d Q,
$$

and

$$
b_{1}=\sum_{\imath=1}^{m} \int_{S_{i}}\left[\alpha_{\imath}(Q)-\beta_{\imath}(Q)\right] d Q
$$




\section{FURTHER RESULTS}

COROLLARY 3.1. Using formulae (14) and (1.5), we deduce as $M \rightarrow \infty$ that

$$
\sum_{\mathrm{k}=1}^{\mathrm{M}}\left[\mu_{k}\left(\alpha_{1}, \ldots, \alpha_{m}\right)-\mu_{k}\left(\beta_{1}, \ldots, \beta_{m}\right)\right]= \begin{cases}{\left[\frac{2 a_{1}}{|\Omega|}+o(1)\right] M} & \text { in the case } n=2, \\ {\left[\frac{2 b_{1}}{V}+o(1)\right] M} & \text { in the case } n=3\end{cases}
$$

Using Theorem 2.2, we easily prove the following theorems:

THEOREM 3.1. Let the functions $\sigma_{\imath}(Q), \alpha_{\imath}(Q), \beta_{\imath}(Q), Q \in \partial \Omega_{\imath}(i=1, \ldots, m)$ and the quantity $a_{1} \neq 0$ be the same as in (2.3) Furthermore, on the half-axis $[c,+\infty)$ let a function $f(\lambda)$ of constant sign be given which is absolutely continuous on each interval $[c, d], d<\infty$; further we assume that the expression $\lambda f^{\prime}(\lambda) / f(\lambda)$ is bounded almost everywhere. Then

(i) If $\int_{c}^{+\infty} f(\lambda) d \lambda=\infty$, we deduce for $\lambda \rightarrow \infty$ that

$$
\begin{array}{r}
\sum_{0<\mu_{k}\left(\sigma_{1}, \ldots, \sigma_{m}\right) \leq \lambda} f\left[\mu_{k}\left(\sigma_{1}, \ldots, \sigma_{m}\right)\right]\left\{\mu_{k}\left(\alpha_{1}, \ldots, \alpha_{m}\right)-\mu_{k}\left(\beta_{1}, \ldots, \beta_{m}\right)\right\} \\
=\left[\frac{a_{1}}{2 \pi}+o(1)\right] \int_{c}^{\lambda} f(t) d t .
\end{array}
$$

(ii) If $\lim _{\lambda \rightarrow \infty} f(\lambda)=\infty$, we deduce for $\lambda \rightarrow \infty$ that

$$
\sum_{0<\mu_{k}\left(\sigma_{1}, \ldots, \sigma_{m}\right) \leq \lambda}\left\{f\left[\mu_{k}\left(\alpha_{1} \ldots, \alpha_{m}\right)\right]-f\left[\mu_{k}\left(\beta_{1}, \ldots, \beta_{m}\right)\right]\right\}=\left[\frac{a_{1}}{2 \pi}+o(1)\right] f(\lambda) .
$$

THEOREM 3.2. Let the functions $\sigma_{\imath}(Q), \alpha_{\imath}(Q), \beta_{\imath}(Q), Q \in S_{\imath}(i=1, \ldots, m)$ and the quantity $b_{1} \neq 0$ be the same as in (2.4). Furthermore, on the half-axis $[c,+\infty)$ let a function $f(\lambda)$ of constant sign be given which is absolutely continuous on each interval $[c, d], d<\infty$; further we assume that the expression $\lambda f^{\prime}(\lambda) / f(\lambda)$ is bounded almost everywhere. Then

(i) If $\int_{c}^{+\infty} \lambda^{1 / 2} f(\lambda) d \lambda=\infty$, we deduce for $\lambda \rightarrow \infty$ that

$$
\begin{aligned}
& \sum_{0<\mu_{k}\left(\sigma_{1}, \ldots, \sigma_{m}\right) \leq \lambda} f\left[\mu_{k}\left(\sigma_{1}, \ldots, \sigma_{m}\right)\right]\left\{\mu_{k}\left(\alpha_{1}, \ldots, \alpha_{m}\right)-\mu_{k}\left(\beta_{1}, \ldots, \beta_{m}\right)\right\} \\
&=\left[\frac{b_{1}}{2 \pi^{2}}+o(1)\right] \int_{c}^{\lambda}|t|^{1 / 2} f(t) d t .
\end{aligned}
$$

(ii) If $\int_{c}^{+\infty} \lambda^{1 / 2} f^{\prime}(\lambda) d \lambda=\infty$, we deduce for $\lambda \rightarrow \infty$ that

$$
\begin{aligned}
\sum_{0<\mu_{k}\left(\sigma_{1}, \ldots, \sigma_{m}\right) \leq \lambda}\left\{f\left[\mu_{k}\left(\alpha_{1}, \ldots, \alpha_{m}\right)\right]-f\left[\mu_{k}\left(\beta_{1}, \ldots, \beta_{m}\right)\right]\right\} \\
=\left[\frac{b_{1}}{2 \pi^{2}}+o(1)\right] \int_{c}^{\lambda}|t|^{1 / 2} f^{\prime}(t) d t .
\end{aligned}
$$

COROLLARY 3.2. Assuming that $f(\lambda)$ of Theorem 3.1(i) has the form $f(\lambda)=\lambda^{j},(j \geq-1)$ then we deduce as $\lambda \rightarrow \infty$ that

$$
\begin{array}{r}
\sum_{0<\mu_{k}\left(\sigma_{1}, \ldots, \sigma_{m}\right) \leq \lambda} \mu_{k}^{\jmath}\left(\sigma_{1}, \ldots, \sigma_{m}\right)\left\{\mu_{k}\left(\alpha_{1}, \ldots, \alpha_{m}\right)-\mu_{k}\left(\beta_{1}, \ldots, \beta_{m}\right)\right\} \\
= \begin{cases}{\left[\frac{a_{1}}{2 \pi(j+1)}+o(1)\right] \lambda^{j+1}} & \text { if } j>-1, \\
{\left[\frac{a_{1}}{2 \pi}+o(1)\right] \ln \lambda} & \text { if } j=-1 .\end{cases}
\end{array}
$$


COROLLARY 3.3. Assuming that $f(\lambda)$ of Theorem 32 (i) has the form $f(\lambda)=\lambda^{\jmath},\left(\jmath \geq-\frac{3}{2}\right)$ then we deduce as $\lambda \rightarrow \infty$ that

$$
\begin{aligned}
\sum_{0 . \mu_{k 11} \ldots \sigma_{\pi_{1}}<\lambda} \mu_{k}^{J}\left(\sigma_{1}, \ldots, \sigma_{m}\right)\left\{\mu_{k}\left(\alpha_{1}, \ldots, \alpha_{m 2}\right)-\mu_{k}\left(\beta_{1}, \ldots, \beta_{m}\right)\right\} \\
\quad= \begin{cases}{\left[\frac{b_{1}}{\pi^{2}(2 \jmath+3)}+o(1)\right] \lambda^{j+\frac{3}{2}}} & \text { if } \jmath>-\frac{3}{2} \\
{\left[\frac{b_{1}}{2 \pi^{2}}+o(1)\right] \ln \lambda} & \text { if } \jmath=-\frac{3}{2} .\end{cases}
\end{aligned}
$$

COROLLARY 3.4. Assuming that $f(\lambda)$ of Theorem 3 l(ii) has the form $f(\lambda)=\lambda^{\jmath},(\jmath>0)$ then we deduce as $\lambda \rightarrow \infty$ that

$$
\sum_{0<\mu_{k}^{\prime}\left(o_{1}, o_{m}\right) \leq \lambda}\left[\mu_{k}^{\jmath}\left(\alpha_{1}, \ldots, \alpha_{m}\right)-\mu_{k}^{\jmath}\left(\beta_{1}, \ldots, \beta_{m}\right)\right]=\left[\frac{a_{1}}{2 \pi}+o(1)\right] \lambda^{\jmath}
$$

COROLLARY 3.5. Assuming that $f(\lambda)$ of Theorem 32 (ii) has the form $f(\lambda)=\lambda^{\jmath},\left(\jmath>-\frac{1}{2}\right)$ then we deduce as $\lambda \rightarrow \infty$ that

$$
\begin{aligned}
& \sum_{0<\mu_{k}\left(\sigma_{1}, \sigma_{m}\right) \leq \lambda}\left[\mu_{k}^{J}\left(\alpha_{1}, \ldots, \alpha_{m}\right)-\mu_{k}^{J}\left(\beta_{1}, \ldots, \beta_{m}\right)\right] \\
& = \begin{cases}{\left[\frac{j b_{1}}{\pi^{2}(2 j+1)}+o(1)\right] \lambda^{j+\frac{1}{2}}} & \text { if } j>-\frac{1}{2}, \\
{\left[\frac{-b_{1}}{4 \pi^{2}}+o(1)\right] \ln \lambda} & \text { if } j=-\frac{1}{2} .\end{cases}
\end{aligned}
$$

COROLLARY 3.6. If $\mu_{k}\left(\beta_{1}, \ldots, \beta_{m}\right) \neq 0$ we deduce from Corollaries 31 and 33 that as $M \rightarrow \infty$

$$
\sum_{k=1}^{M} \frac{\mu_{k}\left(\alpha_{1}, \ldots, \alpha_{m}\right)}{\mu_{k}\left(\beta_{1}, \ldots, \beta_{m}\right)}= \begin{cases}M+\left[\frac{a_{1}}{2 \pi}+o(1)\right] \ln \left(\frac{4 \pi}{|\Omega|} M\right) & \text { if } n=2, \\ M+\left[b_{1}\left(\frac{6}{\pi^{4} V}\right)^{1 / 3}+o(1)\right] M^{1 / 3} & \text { if } n=3\end{cases}
$$

THEOREM 3.3. Let the functions $\sigma_{\imath}(Q), \alpha_{\imath}(Q), \beta_{\imath}(Q) Q \in \partial \Omega_{\imath},(i=1, \ldots, m)$ and the quantity $a_{1} \neq 0$ be the same as in (311). Furthermore, on the half-axis $[c+\infty)$ let a function $f(\lambda)$ of constant sign be given which is absolutely continuous on each interval $[c, d], d<\infty$, further we assume that the expression $\lambda f^{\prime}(\lambda) / f(\lambda)$ is bounded almost everywhere and $\int_{c}^{+\infty} \lambda^{\jmath-1} f(\lambda) d \lambda=\infty(\jmath>0)$ Then as $\lambda \rightarrow \infty$

$$
\begin{array}{r}
\sum_{0<\mu_{k}\left(\sigma_{1}, \sigma_{m}\right) \leq \lambda} f\left[\mu_{k}\left(\sigma_{1}, \ldots, \sigma_{m}\right)\right]\left\{\mu_{k}^{\jmath}\left(\alpha_{1}, \ldots, \alpha_{m}\right)-\mu_{k}^{j}\left(\beta_{1}, \ldots, \beta_{m}\right)\right\} \\
=\left[\frac{a_{1}}{2 \pi} j+o(1)\right] \int_{c}^{\lambda}|t|^{j-1} f(t) d t .
\end{array}
$$

THEOREM 3.4. Let the functions $\sigma_{\imath}(Q), \alpha_{\imath}(Q), \beta_{\imath}(Q) Q \in S_{\imath},(i=1, \ldots, m)$ and the quantity $b_{1} \neq 0$ be the same as in (3.12) Furthermore, on the half-axis $[c,+\infty)$ let a function $f(\lambda)$ of constant sign be given which is absolutely continuous on each interval $[c, d], d<\infty$, further we assume that the 
expression $\lambda f^{\prime}(\lambda) / f(\lambda)$ is bounded almost everywhere and $\int_{c}^{+\infty} \lambda^{j-\frac{1}{2}} f(\lambda) d \lambda=\infty\left(j \geq-\frac{1}{2}\right)$. Then as $\lambda \rightarrow \infty$

$$
\begin{array}{r}
\sum_{0<\mu_{k}\left(\sigma_{1}, \ldots, \sigma_{m}\right) \leq \lambda} f\left[\mu_{k}\left(\sigma_{1}, \ldots, \sigma_{m}\right)\right] \\
=\left[\frac{b_{1}}{2 \pi^{2}} j+o(1)\right] \int_{c}^{\lambda}|t|^{j-\frac{1}{2}} f(t) d t
\end{array}
$$

COROLLARY 3.7. Assuming that $f(\lambda)$ of Theorem 3.3 has the form $f(\lambda)=\lambda^{r}$, where $r$ is a real number. Then as $\lambda \rightarrow \infty$ we get

$$
\begin{array}{r}
\sum_{0<\mu_{k}\left(\sigma_{1}, \ldots, \sigma_{m}\right) \leq \lambda} \mu_{k}^{r}\left(\sigma_{1}, \ldots, \sigma_{m}\right)\left\{\mu_{k}^{j}\left(\alpha_{1}, \ldots, \alpha_{m}\right)-\mu_{k}^{j}\left(\beta_{1}, \ldots, \beta_{m}\right)\right\} \\
=\left\{\begin{array}{lll}
{\left[\frac{j a_{1}}{2 \pi(r+j)}+o(1)\right] \lambda^{r+\jmath}} & \text { if } & r+j>0, \\
{\left[\frac{a_{1}}{2 \pi} j+o(1)\right] \ln \lambda} & \text { if } & r+j=0 .
\end{array}\right.
\end{array}
$$

COROLLARY 3.8. Assuming that $f(\lambda)$ of Theorem 3.4 has the form $f(\lambda)=\lambda^{r}$, where $r$ is a real number. Then as $\lambda \rightarrow \infty$ we get

$$
\begin{array}{r}
\sum_{0<\mu_{k}\left(\sigma_{1}, \ldots, \sigma_{m}\right) \leq \lambda} \mu_{k}^{r}\left(\sigma_{1}, \ldots, \sigma_{m}\right)\left\{\mu_{k}^{j}\left(\alpha_{1}, \ldots, \alpha_{m}\right)-\mu_{k}^{j}\left(\beta_{1}, \ldots, \beta_{m}\right)\right\} \\
= \begin{cases}{\left[\frac{j b_{1}}{\pi^{2}(1+2 \gamma+2 j)}+o(1)\right] \lambda^{r+j+1 / 2}} & \text { if } r+j>-\frac{1}{2}, \\
{\left[\frac{b_{1}}{2 \pi^{2}} j+o(1)\right] \ln \lambda} & \text { if } r+j=-\frac{1}{2} .\end{cases}
\end{array}
$$

COROLLARY 3.9. If $\mu_{k}\left(\beta_{1}, \ldots, \beta_{m}\right) \neq 0$ we deduce from Corollaries 3.7 and 3.8 that as $M \rightarrow \infty$

$$
\sum_{k=1}^{M}\left[\frac{\mu_{k}\left(\alpha_{1}, \ldots, \alpha_{m}\right)}{\mu_{k}\left(\beta_{1}, \ldots, \beta_{m}\right)}\right]^{j}=\left\{\begin{array}{l}
M+\left[\frac{a_{1}}{2 \pi} j+o(1)\right] \ln \left(\frac{4 \pi}{|\Omega|} M\right) \text { if } n=2, \\
M+\left[b_{1}\left(\frac{6}{\pi^{4} V}\right)^{1 / 3} j+o(1)\right] M^{1 / 3} \text { if } n=3
\end{array}\right.
$$

ACKNOWLEDGMENT. The author expresses his grateful thanks to the referee for some interesting suggestions and comments.

\section{REFERENCES}

[1] ZAYED, E.M.E., Some asymptotic spectral formulae for the eigenvalues of the Laplacian, $J$. Austral. Math. Soc. Ser. B, 30 (1988), 220-229.

[2] ZAYED, E.M.E. and YOUNIS, A.I., On the spectrum of the negative Laplacian for general doubly connected bounded domains, Internat. J. Math. \& Math. Sci. 18 (1995), 245-254.

[3] ZAYED, E.M.E., Heat equation for an arbitrary multiply connected region in $\mathbf{R}^{2}$ with impedance boundary conditions, IMA J. Appl. Math., 45 (1990), 233-241.

[4] ZAYED, E.M.E., An inverse eigenvalue problem for an arbitrary multiply connected bounded domain in $\mathrm{R}^{3}$ with impedance boundary conditions, SIAM J. Appl. Math., 52 (1992), 725-729. 


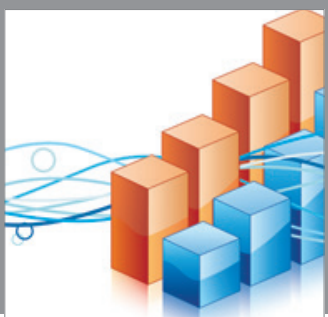

Advances in

Operations Research

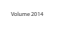

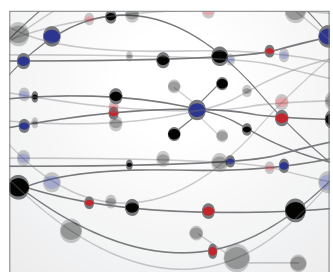

\section{The Scientific} World Journal
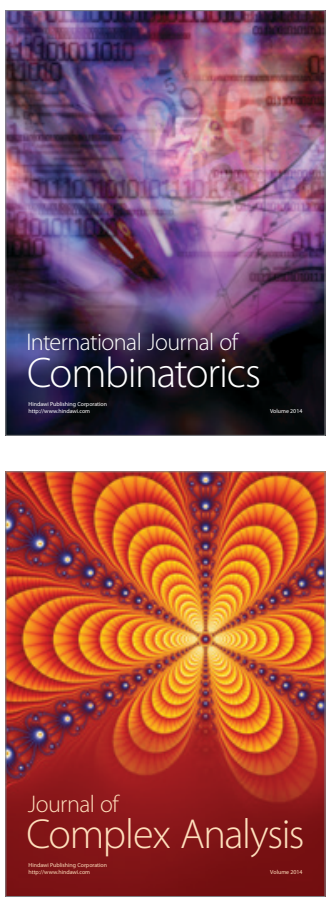

International Journal of

Mathematics and

Mathematical

Sciences
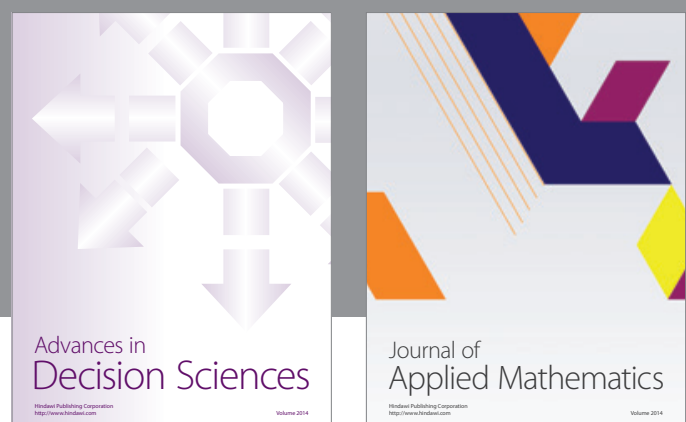

Journal of

Applied Mathematics
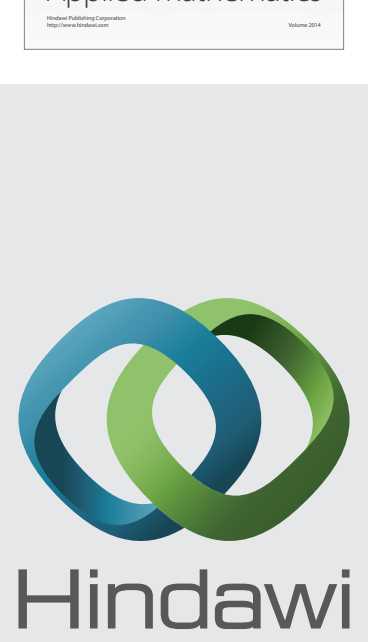

Submit your manuscripts at http://www.hindawi.com
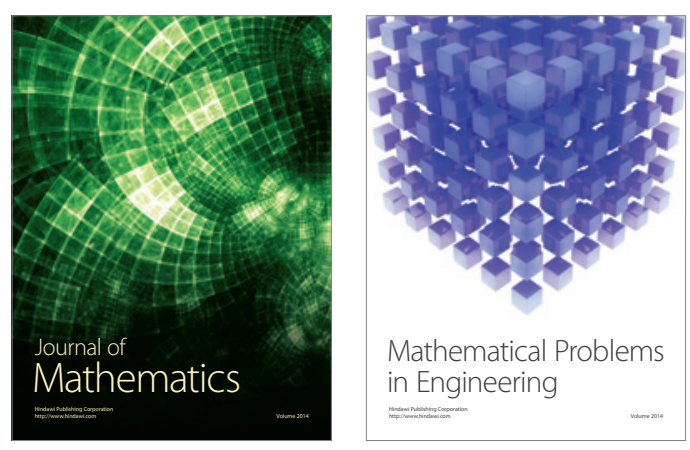

Mathematical Problems in Engineering
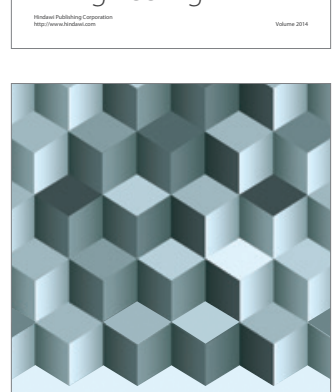

Journal of

Function Spaces
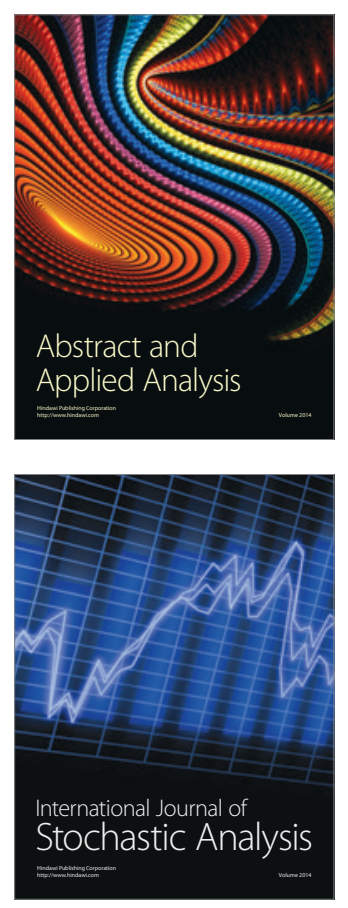

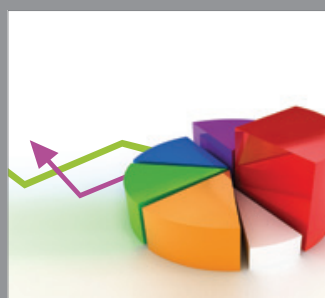

ournal of

Probability and Statistics

Promensencen
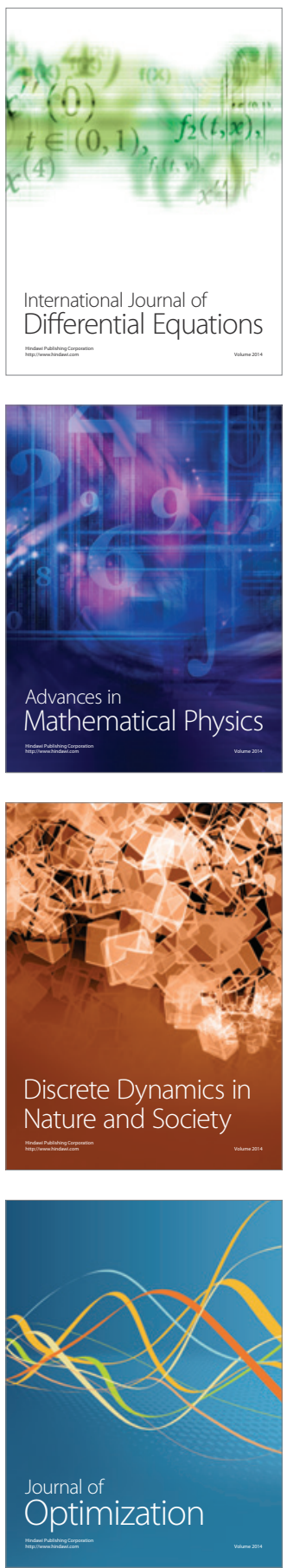